\title{
Detection of MMP-9 and TIMP-3 mRNA expression in the villi of patients undergoing early spontaneous abortion: A report of 30 cases
}

\author{
GUANGLI JIANG and YUXIA QI \\ Department of Obstetrics and Gynecology, Xinxiang Central Hospital, Xinxiang, Henan 453000, P.R. China
}

Received June 8, 2014; Accepted November 28, 2014

DOI: $10.3892 /$ etm.2015.2336

\begin{abstract}
The aim of the present study was to investigate the correlation of matrix metalloproteinase (MMP)-9 and tissue inhibitor of matrix metalloproteinase inhibitor (TIMP)-3 expression with spontaneous abortion (SA) during early pregnancy. The villus tissues of 30 SA cases and 20 requested abortion cases were collected during surgery and constituted the SA and normal abortion (NA) groups, respectively. The total villous RNA was extracted and the expression levels of MMP -9 and TIMP-3 mRNA were detected by reverse transcription-polymerase chain reaction (RT-PCR) assay to calculate the MMP-9/TIMP-3 mRNA ratio. The MMP-9 mRNA expression level and MMP-9/TIMP-3 mRNA ratio of the SA group were significantly higher than those of the NA group ( $\mathrm{P}<0.01)$, while the TIMP-3 mRNA levels of the two groups were similar $(\mathrm{P}>0.05)$. The MMP-9 mRNA expression level of the SA group was higher than that of the NA group; thus, the MMP-9/TIMP-3 mRNA ratio was higher. These results suggest that the expression level of MMP-9 mRNA and the MMP-9/TIMP-3 mRNA ratio are associated with SA.
\end{abstract}

\section{Introduction}

Abortion is defined as termination of pregnancy when the gestation period is $<28$ weeks and the fetal weight is $<1,000 \mathrm{~g}$. An abortion that occurs before 12 weeks of gestation is termed early abortion, accounting for $>80 \%$ of cases of spontaneous abortion (SA). There are numerous causes of abortion, including fetal chromosomal abnormality, maternal systemic disease, genital abnormality, endocrine abnormality, immune

Correspondence to: Dr Yuxia Qi, Department of Obstetrics and Gynecology, Xinxiang Central Hospital, 56 Jinsui Avenue, Xinxiang, Henan 453000, P.R. China

E-mail: yuxiaqicn@163.com

Key words: spontaneous abortion, early pregnancy, matrix metalloproteinase-9, matrix metalloproteinase inhibitor-3, villus, gene dysfunction and environmental factors. In addition, certain unknown reasons for abortion exist (1).

Pregnancy is a successful semi-allograft phenomenon, and embryo implantation is a very complex and sophisticated biological process, which is affected and regulated by a variety of cytokines. Under normal circumstances, the embryo implantation process is similar to the infiltration of tumor cells; the basic biological process is that extravillous cytotrophoblast cells migrate and invade the endometrium (1). In recent years, a number of studies have found that matrix metalloproteinases (MMPs) are associated with invasive diseases and angiogenesis (2-4), and a considerable amount of research has been directed towards the correlations of MMPs with cancer, endometriosis and trophoblastic diseases. MMP family members are inhibited by endogenous tissue inhibitors, namely tissue inhibitors of matrix metalloproteinases (TIMPs). The balance between MMPs and TIMPs determines the invasiveness of cells, and the homeostasis of the two plays a very important role in numerous in vivo physiological and pathological processes. There have been an increasing number of studies concerning the roles of MMPs and TIMPs in the abortion process, some of which have confirmed that in the MMP family, MMP-9 is the major protease involved in the invasion of human cytotrophoblast cells into the endometrium, and plays a major role in the process of embryo implantation and development $(5,6)$. Among the four TIMPs, TIMP-3 is the major tissue inhibitor of MMP-9, which regulates the invasion process of cytotrophoblast cells $(5,6)$. In the present study, the reverse transcription-polymerase chain reaction (RT-PCR) method was used to detect the expression of MMP-9 and TIMP-3 mRNA in the villous tissues of SA patients, with the aim of exploring their correlation with SA.

\section{Materials and methods}

Subjects. A total of 30 SA cases, admitted into the Department of Obstetrics and Gynecology (Xinxiang Central Hospital, Xinxiang, China) from July to December 2013, were selected and defined as the SA group. The patients in this group were aged 23-38 years, with a median age of 29 years. The gestational periods were 56-72 days, with an average of 61 days, and all were confirmed by B-mode ultrasound to have no fetal heart beat. Another 20 patients, who were in normal early pregnancy while requesting an abortion in the same period, were selected 
as the control [normal abortion (NA)] group. The patients in this group were aged 22-44 years, with a median age of 32 years. The gestational periods were 48-68 days, with an average of 58 days; B-mode ultrasound confirmed intrauterine pregnancy and the fetal heart beat could be observed. The two groups were free from genital malformations and infectious diseases, and were not accompanied by surgical and medical complications, genetic diseases and pregnancy complications. There was no statistical significance between the age and gestational age of the two groups $(\mathrm{P}>0.05)$; thus, they were comparable. The present study was approved by the Central Hospital of Xinxiang (Xinxiang, China). Informed consent was obtained from the patients or patients' families prior to their participation.

\section{Methods}

Tissue sampling and processing. Approximately $0.2 \mathrm{~g}$ villi were obtained during uterine curettage, washed with saline, placed into a diethylpyrocarbonate (DEPC)-treated frozen tube, and then stored in liquid nitrogen immediately for further uniform RNA extraction when the specimen collection was completed.

Total RNA extraction. From each sample of villi, 50-80 mg tissue was placed into $1 \mathrm{ml}$ TRIzol RNA reagent (Takara Biotechnology Co., Ltd, Dalian, China) for full homogenization, followed by the addition of $0.2 \mathrm{ml}$ chloroform and centrifugation at $12,000 \mathrm{x} g$ for $15 \mathrm{~min}$. The supernatant was then evenly mixed with an equal volume of isopropanol prior to $10 \mathrm{~min}$ centrifugation at $12,000 \times \mathrm{g}$. The precipitated RNA was then washed with $75 \%$ iced ethanol, and centrifuged at 8,000 x g for $5 \mathrm{~min}$ twice. The supernatant was discarded, while the precipitate was dissolved in 30-100 $\mu 1$ 1\% DEPC solution. A UV spectrophotometer (U-2001; Perkin Elmer, Waltham, MA, USA) was then used to determine the RNA purity and concentration, which was used to control the sample concentration within the range 0.5 to $1.4 \mathrm{~g} / \mathrm{l}$ and the absorbance ratio within the range 1.8 to 2.0.

$R T$ reaction. From each sample, $2 \mu \mathrm{g}$ RNA was extracted, combined with $2 \mu \mathrm{l}$ oligo(dT) $)_{15}$ primer, $4 \mu \mathrm{l}$ 5-fold RT buffer, $1 \mu \mathrm{l}$ Moloney murine leukemia virus reverse transcriptase (M-MLV), $0.5 \mu$ l RNA enzyme inhibition agent and $0.5 \mu 1$ deoxynucleotide triphosphate (dNTP; Shanghai Promega, Shanghai, China), and DEPC was added to provide a final volume of $20 \mu \mathrm{l}$. The reaction conditions were as follows: $70^{\circ} \mathrm{C}$ for $5 \mathrm{~min}$, placement in an ice-water mixture for $5 \mathrm{~min}$, $37^{\circ} \mathrm{C}$ for $5 \mathrm{~min}, 42^{\circ} \mathrm{C}$ for $60 \mathrm{~min}$ and $70^{\circ} \mathrm{C}$ for $15 \mathrm{~min}$. The RT products were then reserved at $-20^{\circ} \mathrm{C}$.

$P C R$. All primers were synthesized by Beijing Huada Jierui Biotechnology Co., Ltd. (Beijing, China) and $\beta$-actin was used as an internal reference. The primer sequences of $\beta$-actin and MMP-9 genes were as described in the literature (3). The TIMP-3 gene primer sequences were automatically designed using Primer Express 3.0 software (Applied Biosystems Life Technologies, Foster City, CA, USA). $\beta$-actin, forward primer: 5'-ACACTGTGCCCATCTATGAGG-3', reverse primer: 5'-GGAGGGGCCGGACTCGTCATACT-3'; MMP-9 gene, forward primer: 5'-GGTGGACCGGATGTTCCC-3', reverse primer: 5'-GCCCACCTCCACTCCTCC-3'; TIMP-3 gene, forward primer: 5'-TCCCTTGGACACTAACTCTTCC-3', reverse primer: 5'-CСТCCCTCACTCTTACATG-3'. The reaction volume was $25 \mu \mathrm{l}: 2.5 \mu \mathrm{l}$ template-complementary DNA (reverse transcription products), $2.5 \mu 1$ 10-fold PCR buffer,
$0.5 \mu 1 \mathrm{dNTP}, 1 \mu \mathrm{l}$ each primer, 0.5 IU Taq DNA polymerase (Shanghai Promega) and DEPC (remainder). The reaction conditions for MMP-9 were initial denaturation at $94^{\circ} \mathrm{C}$ for $5 \mathrm{~min}$, followed by $94^{\circ} \mathrm{C}$ denaturation for $50 \mathrm{sec}, 60^{\circ} \mathrm{C}$ annealing for $50 \mathrm{sec}$ and $72^{\circ} \mathrm{C}$ extension for $50 \mathrm{sec}$, for a total of 30 cycles, and a final extension step at $72^{\circ} \mathrm{C}$ for $5 \mathrm{~min}$. The reaction conditions for TIMP-3 were initial denaturation at $94^{\circ} \mathrm{C}$ for $5 \mathrm{~min}$, followed by $94^{\circ} \mathrm{C}$ denaturation for $50 \mathrm{sec}, 49^{\circ} \mathrm{C}$ annealing for $5 \mathrm{sec}$ and $72^{\circ} \mathrm{C}$ extension for $5 \mathrm{sec}$, for a total of 30 cycles, and a final extension step at $72^{\circ} \mathrm{C}$ for $5 \mathrm{~min}$.

Electrophoresis and semi-quantitative analysis of PCR products. A mixture of $5 \mu \mathrm{l}$ MMP-9 PCR product and $1 \mu 1$ 6 -fold sample buffer was subjected to $1.5 \%$ agarose gel (Shanghai Aybio, Shanghai, China) electrophoresis (80 V) for $30 \mathrm{~min}$. The $5 \mathrm{ul}$ TIMP-3 primer amplification products were added to 1 ul $6 \mathrm{X}$ loading buffer (Beijing GenStar Biosolutions Co., Ltd., Beijing, China), the DNA ladder was set as the reference and $2 \%$ agarose gel electrophoresis $(60 \mathrm{~V})$ was carried out for $60 \mathrm{~min}$. The electrophoresis results were then examined using the Gene Genius gel image analysis system (Syngene Co., Cambridge, UK), with the clear visualization of $28 \mathrm{~S}, 18 \mathrm{~S}$ and $5 \mathrm{~S}$ electrophoresis bands set as the standard, to calculate the levels of target genes. The ratio of the absorbance of the PCR product to that of $\beta$-actin was set as the relative expression level of the target gene mRNA, and the ratio of MMP-9/TIMP-3 mRNAs was calculated.

Statistical analysis. SPSS software, version 10.0 (SPSS, Inc., Chicago, IL, USA) was used for statistical analysis. A t-test of unitizing design was used to compare the results between groups. The measurement data are expressed as the full range (median), and comparisons were conducted using the rank sum test.

\section{Results}

Analysis of MMP-9 and TIMP-3 mRNA expression levels and their ratio in the two groups. The relative expression levels of MMP-9 and TIMP-3 mRNA in the villi of the two groups were determined and the MMP-9/TIMP-3 mRNA ratios were calculated. Intergroup analysis by t-test revealed significant differences in this ratio $(\mathrm{P}<0.001)$. The MMP-9 expression level and MMP-9/TIMP-3 ratio in the SA group were significantly higher than those in the NA group (Table I; Fig. 1). There was no significant difference between the expression level of TIMP-3 in the normal abortion and natural abortion groups.

Agarose gel electrophoresis of MMP-9 and TIMP-3 mRNA expression in the villi of the two groups. The stable expression of $\beta$-actin was detected in all the tissue specimens, confirming the integrity of the cDNA products and the success of the PCR. The fragment length of the $\beta$-actin mRNA RT-PCR product was $621 \mathrm{bp}$, while those of target gene MMP-9 and TIMP-3 mRNAs were 300 bp and 593 bp, respectively (Fig. 2).

\section{Discussion}

The maintenance of normal pregnancy is a synergic process between the embryo and the mother. Cytotrophoblast cells 
Table I. Comparison of the relative expression of MMP-9 and TIMP-3 mRNA in the villi of the two groups.

\begin{tabular}{lcccr}
\hline Group & No. of cases & MMP-9 & TIMP-3 & MMP-9/TIMP-3 \\
\hline SA & 30 & $0.35-0.74(0.55)^{\mathrm{a}}$ & $0.80-1.00(0.90)$ & $0.35-0.80(0.54)^{\mathrm{a}}$ \\
NA & 20 & $0.11-0.39(0.25)$ & $0.76-0.99(0.87)$ & $0.11-0.30(0.19)$ \\
\hline
\end{tabular}

Values presented are the range (median). MMP, matrix metalloproteinase; TIMP, tissue inhibitor of metalloproteinase; SA, spontaneous abortion; NA, normal abortion. $\mathrm{P}<0.01$ compared with the NA group, ${ }^{\text {a }}$.

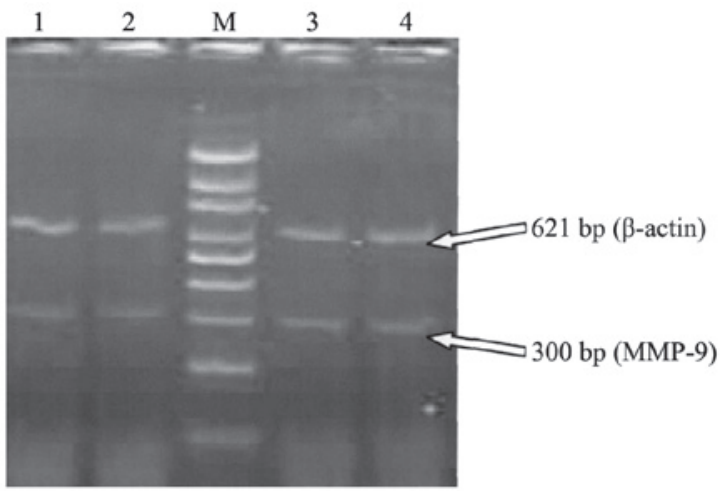

Figure 1. Agarose gel electrophoresis of MMP-9 mRNA expression of the two groups. Lanes 1 and 2, normal early pregnancy; lanes 3 and 4, spontaneous abortion in early pregnancy, Lane M, 100 bp DNA ladder. The samples of villi in lanes 1, 2, 3 and 4 were obtained on the 58th, 60th, 59th and 58th days of pregnancy, respectively. The MMP-9 bands of lanes 3 and 4 were brighter than those of lanes 1 and 2. MMP, matrix metalloproteinase.

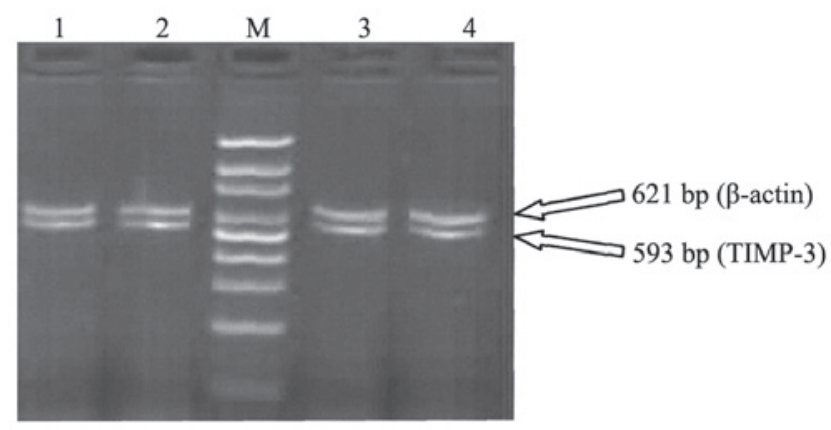

Figure 2. Agarose gel electrophoresis of TIMP-3 mRNA expression of the two groups. Lanes 1 and 2, normal early pregnancy; lanes 3 and 4, spontaneous abortion in early pregnancy, Lane M, 100 bp DNA ladder. The samples of villi in lanes 1, 2, 3 and 4 were obtained on the 58th, 60th, 59th and 58th days of pregnancy, respectively. The brightness of the TIMP-3 bands in lanes 1-4 were similar to each other. TIMP, tissue inhibitor of metalloproteinase.

in normal early pregnancy have similar characteristics to malignant cells. They secrete a large amount of MMPs, thus selectively hydrolyzing the endometrial stromal components and basement membrane, while simultaneously generating TIMPs to inhibit MMP generation. This maintains the dynamic balance of MMPs/TIMPs within a certain range, and subjects the invasion of cytotrophoblast cells into the maternal body to certain restrictions $(5,6,7)$. The majority of non-Chinese studies have shown that MMP-9 and TIMP-3 play particularly important roles in early pregnancy; the MMP-9/TIMP-3 balance is significant in regulating the depth of invasion of cytotrophoblast cells into the uterus and embryo implantation $(8,9)$. There have also been certain Chinese studies indicating that the high expression levels of TIMP-3 during the endometrial implantation window may avoid the excessive degradation of endometrial extracellular matrix (ECM) by MMP-9, prevent the excessive infiltration of cytotrophoblast cells, and regulate the activity of MMP-9. An imbalance in the expression of MMP-9 and TIMP-3 would affect fetal growth and development, and excessive invasion by cytotrophoblast cells is likely to result in miscarriage in early pregnancy (7).

Among the various MMPs that are secreted by the maternal endometrium and cytotrophoblast cells during the processes of endometrial degeneration and blastocyst implantation, MMP-9 is considered to be the main proteolytic enzyme (10). MMP-9 is a major proteolytic enzyme, the activity of which is dependent on $\mathrm{Zn}^{2+}$ and $\mathrm{Ca}^{2+}$, and it is the main enzyme that degrades the ECM. During the invasion of placental cytotrophoblast cells into the maternal uterine stroma, type IV collagen is the main component of the ECM between the fetus and the mother, and the substrate that MMP-9 decomposes is type IV collagen. Following implantation, MMP-9 decomposes the ECM, and initiates cytotrophoblast adhesion, migration and differentiation; thus, cytotrophoblast cells are able to penetrate the basement membrane and enter the maternal circulation. During the in vitro cultivation of cytotrophoblast cells, Whiteside et al (5) found that the cells secreted a large amount of MMP-9 and that MMP-9 expression was significantly increased subsequent to embryo implantation, which would be beneficial towards embryo implantation and placental formation. Jeziorska et al (11) measured MMP-9 and MMP-3 secretion every day of the menstrual cycle, and revealed that MMP-9 expression was regulated precisely and according to the time within the cycle; MMP-9 was not expressed in endometrial tissues in the early proliferation stage, began to exhibit glandular epithelial expression on day 7 of the cycle, with extracellular secretion into the glandular lumen appearing from day 15. On days 21-23, the expression reached a peak and was also observed in the uterine cavity, which lasted until menstruation. MMP-9 is richly expressed in the post-ovulation implantation window, suggesting that it is conducive to blastocyst implantation. $\mathrm{Xu}$ et al (12) performed in vitro culture of human cytotrophoblast cells for 6-11 weeks. At the end of week 6, no MMP-9 secretion was detected, while secretion started and gradually increased from weeks 7-11, and reached a level in week 11 that was 10 -fold greater than that in week 7. By contrast, the expression of MMP-2 decreased from week 6 to week 11, and the amount expressed in week 11 was only one-sixth of that in week 6 . The expression levels of MMP-2 and MMP-9 mRNA were 
consistent with their protein secretion, suggesting that MMP-2 plays an important role in the human embryo peri-implantation period. After that, the invasive ability of cytotrophoblast cells is likely to be determined by MMP-9 production. MMP-9 was also detected in the chorionic villi in the present study, suggesting that MMP-9 plays an important role in the process of cytotrophoblast invasion. Further experiments found that MMP-9 mRNA expression levels in the SA patients were significantly higher than those of normal early pregnancy $(\mathrm{P}<0.01)$, which may be due to the cytotrophoblast cells of SA patients producing excessive quantities of MMP-9, thus reinforcing the hydrolysis of basement membrane proteins, and resulting in the excessive invasion, infiltration and metastasis of cytotrophoblast cells.

In the TIMP family of proteins, TIMP-3 has been shown to be the main inhibitor of MMP-9. A study by Whiteside et al (5) found that MMP-9 was expressed by embryonic cytotrophoblast cells, and that when an MMP-9 antisense oligonucleotide was used to treat mouse blastocysts cultivated on an ECM gel, ECM degradation by the mouse blastocysts was significantly reduced due to restricted MMP-9 protein secretion. In TIMP-3-enriched ECM, the degradation of ECM by growing blastocysts was also reduced compared with that of normal ECM, in a dose-dependent manner; this decline was due to the inhibition of MMP-9 activity by TIMP-3. This suggests that interaction between MMP-9 and TIMP-3 is the key mechanism by which cytotrophoblast cells implement an appropriate invasive force, and explains the ability of cytotrophoblast cells to avoid excessive invasion towards the endometrium. The present study detected the expression of TIMP-3 mRNA in the villi of normal early pregnancy, and the results revealed that the TIMP-3 mRNA expression levels in the SA patients and patients with normal pregnancy were equivalent $(\mathrm{P}>0.05)$.

During the in vitro cultivation of cytotrophoblast cells, Whiteside et al (5) found that the cytotrophoblast cells secreted large amounts of MMP-9 and that MMP-9 expression began to increase significantly subsequent to implantation, indicating that it was favorable for embryo implantation and placental formation. Polette et al (13) also observed that MMP-9 was secreted at the highest levels in early pregnancy. Successful embryo implantation requires a suitable intrauterine environment and receptivity; as it has been found that the MMP-9 activities in the endometria of women with failed early pregnancy are significantly increased and result in disorders of ECM components, this suggests that MMP-9 is significant in the failure of pregnancy (14). The present study also detected MMP-9 in the chorionic villi of women in the early stages of pregnancy, suggesting that MMP-9 plays an important role in cytotrophoblast invasion. Table I demonstrates that the MMP-9 expression level of the SA group was significantly increased compared with that in the NA group $(\mathrm{P}<0.001)$, indicating that the chorionic cytotrophoblast cells of the SA group produced excessive amounts of MMP-9. Thus, the proteolytic effects of MMP-9 towards the endometrial basement membrane were increased, and were significant in the invasion, infiltration and metastasis of cytotrophoblast cells. These results are consistent with those of Iwahashi and Nakano (15).

In a study of rhesus monkeys, MMP-9 was found to be a critical factor for cytotrophoblast invasion into the endometrium and placenta formation; however, it was also detected that TIMP-3 mRNA was highly expressed in certain endo- metrial arteries, suggesting that it exhibited an important role in preventing decidual bleeding and abortion (6). Chinese research has demonstrated that the synergic expression of MMP-9 and TIMP-3 is necessary to maintain normal early pregnancy $(15,16)$. The synergy of MMP-9 and TIMP-3 is very important in regulating the cytotrophoblast invasion depth in the uterus and embryo implantation $(7,8,17)$. Whiteside et al (5) reported that the expression levels of embryo-derived MMP-9 and ECM-derived TIMP-3 were correlated. Increased MMP-9 expression was accompanied by high TIMP-3 expression, which antagonized the degradation effect of MMP-9 on the ECM, and avoided the excessive invasion of cytotrophoblast cells into the endometrium. A stable dynamic equilibrium between these two proteins enabled the pregnancy to proceed smoothly. Li et al (7) reported when MMP-9 is dominant, it promotes ECM degradation, which is profitable for embryo implantation with the continuous invasion of cytotrophoblast cells and when TIMP-3 is dominant, it inhibits the excessive degradation of ECM by MMP-9 and prevents the excessive invasion of villous cytotrophoblast cells; therefore, the balance of these two proteins may help to maintain normal pregnancy. In the present study, the ratio of MMP-9/ TIMP-3 mRNA expression in the SA group was significantly higher than that in the NA group $(\mathrm{P}<0.01)$. However, with the increased expression of MMP-9, the TIMP-3 level of the SA group did not increase appropriately to antagonize the ECM-degrading effect of MMP-9. This resulted in an increase in the MMP-9/TIMP-3 mRNA ratio of the SA group. From these results, it may be speculated that the synergic expression of MMP-9 and TIMP-3 genes has a close association with the depth of invasion of cytotrophoblast cells. The imbalance in the levels of MMP-9 and TIMP-3 is likely to cause excessive degradation of the ECM and the excessive invasion of cytotrophoblast cells into the endometrium, thus affecting embryo implantation and even resulting in pathological SA or other pathological pregnancy.

In summary, the MMP-9 mRNA expression levels of the patients undergoing SA were high compared with those in normal patients, and the MMP-9/TIMP-3 mRNA ratio was also high in the SA group. The MMP-9 mRNA expression level and MMP-9/TIMP-3 mRNA ratio may be relevant to SA. The results of this study provide an experimental basis for the clinical investigation of the mechanism of abortion, and also a theoretical basis for the clinical application of MMP-9 as a diagnostic indicator of threatened abortion. In addition, they support the use of synthetic TIMPs to treat threatened abortion and maintain a normal pregnancy.

\section{References}

1. Xie X: Abnormal pregnancy Section I: spontaneous abortion. In: Science of Obstetrics and Gynecology. Gou W (ed). 8th edition. People's Medical Publishing House, Beijing, China, pp47, 2013.

2. Itoh T, Tanioka M, Matsuda H, et al: Experimental metastasis is suppressed in MMP-9-deficient mice. Clin Exp Metastasis 17: 177-181, 1999.

3. Huang S, Van Arsdall M, Tedjarati S, et al: Contributions of stromal metalloproteinase- 9 to angiogenesis and growth of human ovarian carcinoma in mice. J Natl Cancer Inst 94: $1134-1142,2002$

4. Okamoto T, Niu R, Yamada S and Osawa M: Reduced expression of tissue inhibitor of metalloproteinase (TIMP)-2 in gestational trophoblastic diseases. Mol Hum Reprod 8: 392-398, 2002. 
5. Whiteside EJ, Jackson MM, Herington AC, et al: Matrix metalloproteinase- 9 and tissue inhibitor of metalloproteinase- 3 are key regulators of extracellular matrix degradation by mouse embryos. Biol Reprod 64: 1331-1337, 2001.

6. Wang H, Li Q, Shao L and Zhu C: Expression of matrix metalloproteinase-2, $-9,-14$ and tissue inhibitors of metalloproteinase-1, $-2,-3$, in the endometrium and placenta of rhesus monkey (Macaca mulatta) during early pregnancy. Biol Reprod 65: 31-40, 2001.

7. Li L, Xing FQ and Chen SL: Regulation of TNF- $\alpha$ towards the expression of endometrial metalloproteinase- 9 and its tissue inhibitor 3. Zhonghua Fu Chan Ke Za Zhi 41: 346-347, 2006 (In Chinese).

8. Walter I and Schönkypl S: Extracellular matrix components and matrix degrading enzymes in the feline placenta during gestation. Placenta 27: 291-306, 2006.

9. LaMarca HL, Ott CM, Höner Zu Bentrup K, et al: Threedimensional growth of extravillous cytotrophoblasts promotes differentiation and invasion. P1acenta 26: 709-720, 2005.

10. Nuttall RK and Kennedy TG: Gelatinases A and B and tissue inhibitors of metalloproteinases 1,2 and 3 during in vivo and in vitro decidualization of rat endometrial stromal cells. Biol Reprod 60: 471-478, 1999.

11. Jeziorska M, Nagase H, Salamonsen LA and Woolley DE: Immunolocalization of the matrix metalloproteinase gelatinase $\mathrm{B}$ and stromelysin 1 in human endomentrium throughout the menstrual cycle. J Reprod Fertil 107: 43-51, 1996.
12. Xu P, Wang YL, Zhu SJ, Luo SY, Piao YS and Zhuang LZ: Expression of matrix metalloproteinase $-2,-9$, and-14, tissue inhibitors of metalloproteinase-1, and matrix proteins in human placenta during the first trimester. Biol Reprod 62: 988-994, 2000.

13. Polette M, Nawrocki B, Pintiaux A, et al: Expression of gelatinases $\mathrm{A}$ and $\mathrm{B}$ and their tissue inhibitors by cells of early and term human placenta and gestational endometrium. Lab Invest 71: 836-846, 1994

14. Inagaki N, Stern C, McBain J, et al: Analysis of intra-uterine cytokine concentration and matrix-metalloproteinase activity in women with recurrent failed embryo transfer. Hum Reprod 18: 608-615, 2003.

15. Iwahashi M and Nakano R: Decreased type V collagen expression in human decidual tissues of spontaneous abortion during early pregnancy. J Clin Pathol 51: 44-46, 1998.

16. Bass KE, Li H, Hawkes SP, Howard E, Bullen E, Vu TK, et al: Tissue inhibitor of metalloproteinase-3 expression is upregulated during human cytotrophoblast invasion in vitro. Dev Genet 21: 61-67, 1997.

17. Gui W, Qiu Y, Zeng P, et al: Expression of matrix metalloproteinase- 9 and tissue inhibitor of metalloproteinase-I protein in the implantation of window phase of endometrium in ovulation normal women. Chongqing Yike Daxue Xuebao 29: $1-6,2004$. 